\title{
Comparative Analysis of Parasitological, Immunodiagnostic and Histopathological Methods in the Diagnosis of Human Giardiasis
}

\author{
NATAŠA MILADINOVIĆ TASIĆ ${ }^{*}$, VUKA KATIĆ ${ }^{2}$ \\ ${ }^{1}$ University of Niš, Faculty of Medicine, Niš, Serbia Public Health Institute, Niš, Serbia \\ ${ }^{2}$ Polyclinic Human, Niš, Serbia
}

\begin{abstract}
Giardiasis/lambliosis is a disease caused by the presence of a protozoan Giardia lamblia (G. lamblia) in the upper portions of the human digestive tract (DT). This cosmopolitan parasite is present particularly in children, but in all other age groups and socioeconomic conditions as well. The studies undertaken in the Nišava District (southeastern Serbia) have demonstrated a high prevalence of infection caused by G. lamblia species using conventional microscopy of three stool samples with or without concentration technique (6.24\%), i.e. using the immunoenzymatic method to confirm the presence of G. lamblia antigen in the stool (6.59\%). The use of invasive methods in the diagnosis of giardiasis is warranted in individual selected cases, when the significance of the parasite should be assessed regarding its involvement in the pathogenetic mechanisms of damage to the digestive tract mucosa.
\end{abstract}

Keywords: gardiasis, convencional microscopy, immunoenzymatic method, invasive methods

Giardiasis/lambliosis is a disease caused by the presence of a protozoan Giardia lamblia (G. lamblia) in the upper portions of the human digestive tract (DT). Infectious cysts may enter the organism via contaminated water, food, or by direct fecal-oral contact. A single cyst is all it takes for the infection to occur, although the studies on volunteers have showed that 10-25 cysts are usually required. After the incubation period of 7-10 days on the average, the disease symptoms appear, which are diverse. However, most of the patients infected with the protozoan are asymptomatic.

Conventional microscopy (CM) of three stool samples with or without concentration technique is still recommended as the reference method (the ,gold standard") in the diagnosis of the DT infection caused by $G$. lamblia. With the introduction of new diagnostic methods, demonstration of antigen presence in the feces using enzyme immunoabsorption assays (EIA), nonenzymatic assays, and/or fluorescein-labeled monoclonal antibodies, the diagnosis of giardiasis has been significantly improved, especially in the assessment of disease course and screening of G. lamblia infection in epidemiological studies [1- 6]. In patients in whom parasitological and immuno-diagnosis has not been made, an endoscopy with duodenal fluid sampling and biopsy of the duodenal or gastric mucosa can be performed $[7,8]$.

Culturing and sensitive tests using the molecular methods such as DNA probes and PCR are available mostly in research centers and are not used in everyday, routine work. DNA probes are usually used to demonstrate the presence of parasites in water samples, while PCR is commonly used in molecular epidemiology and examination of the zoonotic and pathogenic potentials of G. lamblia [9-13].

\section{Experimental part}

Material and methods

$\underline{\text { Patients }}$

The study involved 110 adults with digestive tract complaints (nausea, urge to vomit, abdominal pain, occasional diarrhea, etc.). Of the total number of cases, $74(67,27 \%)$ patients aged on the average $45 \pm 17,39$ years (min 16 , $\max$ 78 years; 43 women and 31 men) were referred to the Parasitology Laboratory by their primary care physicians, while 36 persons aged on the average $62 \pm 14,41$ years ( $\min 22$, max 77 years; 19 men $(19 / 36 ; 52,77 \%$ ) and 17 women $(17 / 36 ; 47,22 \%))$ were hospitalized for: i) recurrent digestive tract infection in spite of the administered therapy; ii) chronic digestive tract infections with severe symptoms and without any detected abnormalities on bacterology and mycology examination of the stool; and iii) primary diseases accompanied by digestive tract (DT) complaints.

\footnotetext{
*email: nmiltasic@yahoo.com
} 
Parasitological examinations

Parasitological diagnosis of G. lamblia in the stool was performed using conventional microscopy (CM) of three consecutive samples (1-3 days apart, for the period of 10 days), with or without concentration methods (formalin-ether method by Ritchie) [14]. The samples of fresh, liquid stool were examined in the period of 30 minutes after the receipt in the lab, examining the stained (Lugol solution and fluorescent staining with acridine orange) and/or unstained native preparation (NP), while the samples of formed stool were processed and examined in the period of 2 hours of the receipt in the lab. In absence of any accurate standard, CM served as the gold standard.

\section{Immunodiagnostic examinations}

Immunodiagnosis from a single stool sample, using a commercial enzyme immunoassay (EIA) (Ridascreen Giardia; R-Biopharm, Germany) to establish G. lamblia antigen in the stool, was performed in all examinees, abiding by the manufacturer's instructions. In cases in which the values near the cut-off (borderline) resulted, an additional analysis from the second stool sample was done.

\section{Invasive examinations (endoscopy and biopsy)}

In hospitalized patients (HP), for diagnostic purposes only, duodenoscopy and biopsy of duodenal and gastric mucosa were performed using the standard procedures. Sampling of the duodenal juice and examination of the presence of G. lamblia trophozoites was performed, and then histopathological examination of the DT (primarily duodenal) mucosal biopsies followed.

Duodenal juice, half an hour after sampling at the latest, was prepared for parasitological analysis, and the tissue obtained by biopsy was histopathologically processed. Paraffin sections were stained utilizing the classical hematoxylin-eosin (HE) method, as well as the following histochemical methods: Trichrome staining (Gomori) to demonstrate the G. lamblia protozoan in the sampled tissue, and Alcian blue, ph 2.5-Periodic acid-Schiff (AB-PAS) for histochemical confirmation of the degree of duodenitis and gastritis, but also to demonstrate the G. lamblia protozoan in the sampled tissue.

Gastroduodenitis was classified according to Whitehead [15], and in gastritis staging the types of metaplasia were taken into account, primarily intestinal metaplasia (IM) and pyloric metaplasia (PM). Gastritis activity was determined based on the presence of neutrophil leukocytes in the dermis and crypts, which was designated as active gastritis [15, 16].

Epithelial mucins in the gastric and duodenal mucosa are the characteristic marker of the degree of inflammation; type of epithelial metaplasia; and degree of dysplasia. The division of mucins into fucomucins (neutral mucins), syalomucins (weakly acidic mucins), and sulphomucins (strongly acidic mucins) was used [17].

\section{$\underline{\text { Statistical analysis }}$}

Data entry and analysis were performed using the Epi Info (Ver.6.04) software and SPSS (Ver. 8.0 for Windows). The results of $p<0.05$ were considered statistically significant.

\section{Results and discussions}

The presence of $G$. lamblia was established in 7 out-patients using the CM and EIA. Negative findings of $G$. lamblia in the feces were obtained in $67(67 / 74 ; 90.54 \%)$ patients using both methods. Using CM in $6(6 / 7 ; 85.71 \%)$ patients and NP examination (without a concentration method) G. lamblia cysts were found already in the first stool sample. In the second sample, using NP examination without a concentration method, G. lamblia cysts were established in 4, and after a concentration method in 6 patients. In the third stool sample, the parasite was found in 5 patients using NP examination without a concentration method, and after a concentration method the presence of $G$. lamblia was confirmed in 7 patients.

Using the EIA method, G. lamblia antigen was confirmed in the first stool sample of all 7 patients (Table 1).

Table 1

POSITIVE STOOL SAMPLE FINDINGS AND METHODS USED IN THE DIAGNOSIS OF GIARDIASIS IN OUT-PATIENTS

\begin{tabular}{|c|c|c|c|c|c|c|c|c|}
\hline \multirow{3}{*}{ Patients } & \multicolumn{8}{|c|}{ Methods used in the diagnosis of G. lamblia infection and number of samples } \\
\hline & \multirow{2}{*}{ ELA $^{a}$} & \multirow{2}{*}{$\mathrm{CM}^{\mathrm{b}}$} & \multicolumn{3}{|c|}{ NPo } & \multicolumn{3}{|c|}{ NPkd } \\
\hline & & & $\mathrm{I}$ & II & III & $\mathrm{I}$ & II & III \\
\hline 1. & + & + & $\Psi$ & + & + & $\boldsymbol{H}$ & + & + \\
\hline 2. & + & + & $\Psi$ & + & + & $\Psi$ & + & + \\
\hline 3. & + & + & $\Psi$ & - & + & $\Psi$ & + & + \\
\hline 4. & + & + & $\Psi$ & - & + & $H$ & + & + \\
\hline
\end{tabular}




\begin{tabular}{|c|c|c|c|c|c|c|c|c|}
\hline 5. & + & + & $\boldsymbol{H}$ & + & + & $\boldsymbol{H}$ & - & + \\
\hline 6. & + & + & $\mathbf{H}$ & + & - & $\mathbf{H}$ & + & + \\
\hline 7. & + & + & - & - & - & - & $\mathbf{H}$ & + \\
\hline $\begin{array}{c}\text { Total } \\
\text { positive }\end{array}$ & 7 & 7 & 6 & 4 & 5 & 6 & 6 & 7 \\
\hline
\end{tabular}

${ }^{a} \mathrm{EIA}$, immunoassay method; ${ }^{b} \mathrm{KVM}$, conventional microscopy; ${ }^{c} \mathrm{NP}$, native preparation without a concentration method; ${ }^{d} \mathrm{NPk}$, native preparation after a concentration method

Using the fluorescent AO staining method (Figure 1), positive findings of G. lamblia were obtained as well in the samples taken from the same patients.
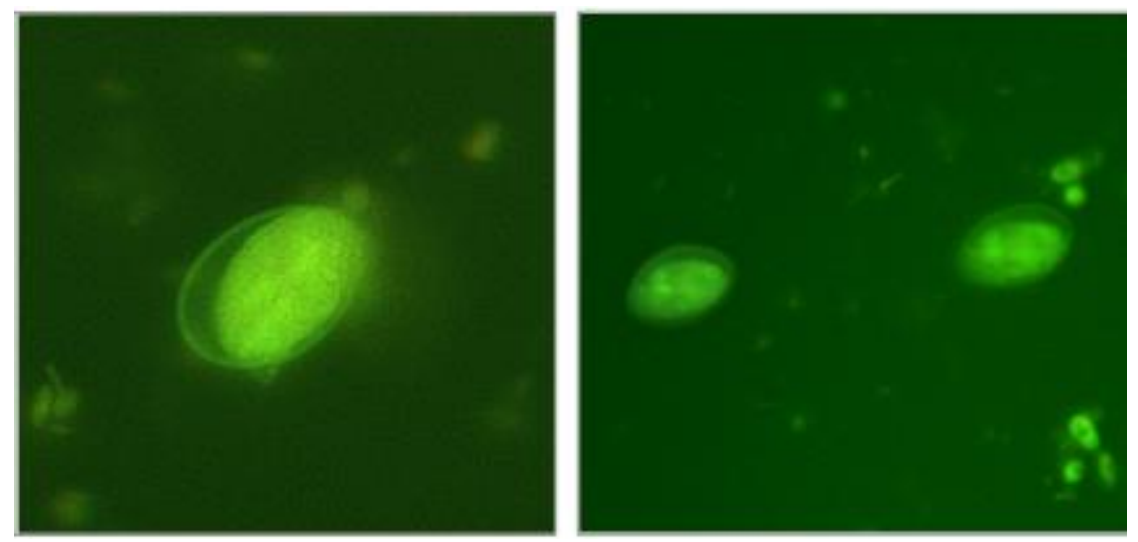

Fig. 1. G. lamblia cysts, direct AO stained preparations $(\times 600, \times 400)$ [N.M.Tasić]

In all 7 patients, the signs of acute giardiasis were evident (nausea, epigastric pain, urge to vomit, diarrhea lasting more than three days), but with variable severity and periods of occurrence (from January to May, as well as in July and August), in different situations and exposure to risk factors.

Our hospitalized patients (HP) had variable complaints. The most common diagnosis found in the studied patients was duodenal ulcer $(15 / 36 ; 41.7 \%)$, but the protozoan of interest was not found in them (Table 2). Among the patients with dyspeptic complaints $(6 / 36 ; 16.7 \%)$ G. lamblia was detected in $4(4 / 36 ; 11.11 \%)$.

Table 2

THE NUMBER OF HPS BY CLINICAL ENTITIES

\begin{tabular}{|c|c|}
\hline Number of patients (36) & Clinical entities \\
\hline $6(16,7 \%)$ & Dispeptic syndrome \\
\hline $4(11,1 \%)$ & Gastric ulcer \\
\hline $15(41,7 \%)$ & Duodenal ulcer \\
\hline $2(5,5 \%)$ & Postgastrectomy \\
\hline $1(2,8 \%)$ & Gastric erosion \\
\hline $1(2,8 \%)$ & Chronic pancreatitis \\
\hline $4(11,1 \%)$ & Chronic diarrhea \\
\hline $3(8,3 \%)$ & \\
\hline
\end{tabular}

In HPs, 108 stool samples were examined using CM, while the presence of $G$. lamblia antigen was examined using EIA in 36 stool samples. The same number of duodenal juice specimens were examined, and as for histopathology, 28 gastric mucosa samples and 36 duodenal biopsy samples were processed. Using the CM and EIA methods, G. lamblia was found in 4 patients $(4 / 36 ; 11.1 \%)$. Examination of the duodenal juice confirmed the presence of parasites in 2 patients, and examination of HP preparations of gastric and duodenal biopsy established the presence of parasites in 1 patient (Table 3). 
Table 3

POSITIVE FINDINGS IN HPS RELATED TO GENDER AND METHODS USED IN G. lamblia DETECTION

\begin{tabular}{|c|c|c|c|c|c|}
\hline \multirow{2}{*}{ Patients } & \multirow{2}{*}{ Gender } & \multicolumn{4}{|c|}{ Methods used in the diagnosis of G. lamblia infection } \\
\cline { 3 - 6 } & & EIA $^{2}$ & $\mathrm{CM}^{2}$ & $\mathrm{DJ}^{2}$ & HPM $^{2}$ \\
\hline 1. & $\mathrm{~F}^{2}$ & + & + & + & + \\
\hline 2. & $\mathrm{M}$ & + & + & - & - \\
\hline 3. & $\mathrm{M}$ & + & + & - & - \\
\hline 4. & $\mathrm{M}$ & + & + & + & 1 \\
\hline \multicolumn{2}{|r|}{ Total positive } & 4 & 4 & 2 & + \\
\hline
\end{tabular}

Trophozoites of $G$. lamblia were confirmed in the duodenal juice in $2(2 / 4 ; 50 \%)$ patients in whose stool samples the presence of the studied parasite was confirmed using CM and EIA methods. In another 2 patients, duodenal juice examination produced a negative G. lamblia result, while CM and EIA stool sample examination produced positive findings. In only one patient, the studied parasite was confirmed using all the above methods. Positive microscopic findings were positive on $\mathrm{AO}$ staining as well. The diagnosis of lambliosis was confirmed in all 4 patients using the EIA method, while the analysis of native preparations from the first and second sample and without a concentration method the presence of G. lamblia was established.

Histopathological examination involved processing of 28 samples of gastric and 36 samples of duodenal mucosa. In all 28 gastric mucosa samples, the presence of different grades of gastritis was found. In most of the cases (18/28; 64.28\%) mild gastritis was established (superficial chronic gastritis, SHG, and the first grade of chronic atrophic gastritis, HAG), while the second and third grade gastritis was established in another $10(10 / 28 ; 35.71 \%)$ patients). Active form of gastritis was established in $11(11 / 28 ; 39.28 \%)$ patients, metaplasia of the gastric mucosa in 4 persons $(4 / 28 ; 14.28 \%)$, while dysplasia was present in 2 patients $(2 / 28 ; 7.14 \%)$.

In the histological preparations of 36 biopsies of the duodenal mucosa, superficial duodenitis was seen in $8(8 / 36$; 22.22\%), i.e. chronic atrophic duodenitis (HAD) in 28 patients (I grade HAD in 10/36, 27.77\%; II grade HAD in $16 / 36,44.44 \%$; and III grade HAD in 2/36, 5.55\% patients). In 15 patients (15/36; 41,66\%), neutrophil infiltration was established as well, i.e. an active disease phase. Metaplasia was present in 14 samples of duodenal mucosa biopsy material $(14 / 36 ; 38.89 \%)$.

Based on the epithelial mucins in gastric and duodenal mucosa the degree of inflammation was established, as well as the type of epithelial metaplasia and degree of dysplasia. The established hyposecretion of epithelial mucins was proportional to the atrophic inflammation and ranged from hypo- to asecretion; it was also an important property of the degree of dysplasia. Histochemistry of epithelial mucins confirmed pyloric metaplasia in chronic duodenitis and intestinal metaplasia in chronic gastritis.

Hisopathological examination of endoscopic biopsies revealed G. lamblia in one duodenal mucosa sample (Fig. 2). In a female patient aged 23 years, II grade chronic atrophic duodenitis was found in active disease phase, and vegetative forms of $G$, lamblia parasite were found in the mucus. Other diagnostic methods used in this patient confirmed as well G. lamblia positivity.

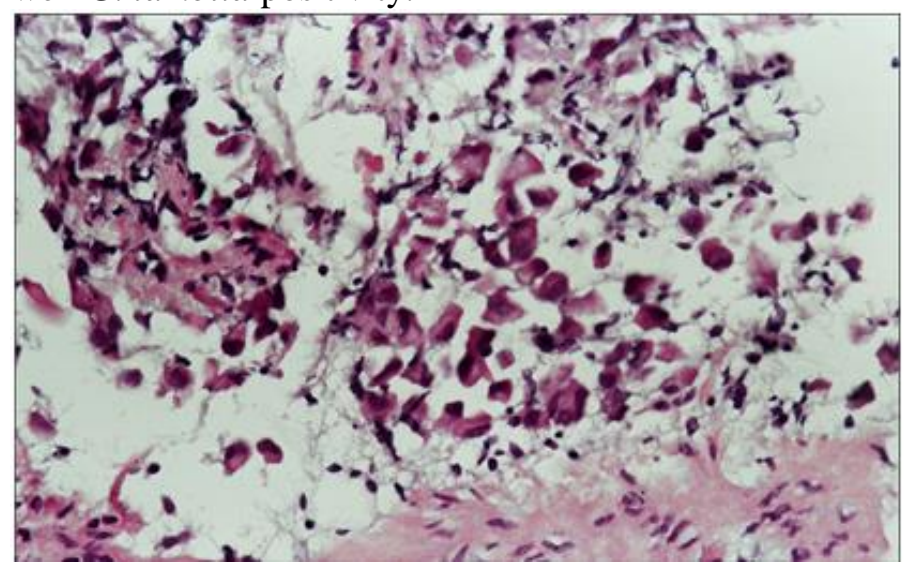

Fig. 2. Vegetative forms of $G$. lamblia in the mucus of duodenal mucosa

The results confirming G. lamblia infection in adults with symptomatic lambliosis using CM and EIA methods were identical: the parasite was established in 11 out of 110 examined patients, so that the prevalence of G. lamblia infection was $10 \%(11 / 110 ; 95 \% \mathrm{CI}=4.37-15.63)$. The presence of $G$. lamblia was confirmed in 7 examined outpatients (the value of prevalence 9.46\%; 7/74; 95\% CI=2.75-16.17), i.e. in the group of hospitalized patients $G$. lamblia was confirmed in 4 out of 36 examined patients (the value of prevalence $11.11 \% ; 4 / 36 ; 95 \% \mathrm{CI}=0.70-27.52$ ). 
Statistical data processing did not reveal any significant gender-related differences in prevalence (6 men/5 women), either in out-patient examinees ( $3 \mathrm{men} / 4$ women) or in hospitalized adults ( $3 \mathrm{men} / 1$ woman). Reviews of the literature data dealing with the prevalence of giardiasis related to the factor of gender, did not reveal any significant differences $[17,18]$.

Introduction of the EIA tests in the diagnosis of parasitic infections is a turning point in the diagnosis of giardiasis. In addition to the comparison of different numbers of samples using $\mathrm{CM}$ and commercial tests based on the concentration method, numerous comparative examinations of the EIA efficacy have been done as well in different groups of interest, depending on the presence or absence of symptoms and signs of infection of the DT [1-6, 19]. The obtained results and their evaluation showed that EIA is a satisfactory alternative to CM in the diagnosis of symptomatic giardiasis, except in cases of negative results, when $\mathrm{CM}$ of a larger number of samples could help resolve the dilemma if the patient had been infected with G. lamblia protozoan.

If the mentioned diagnostic procedures cannot provide positive findings of G. lamblia, many authors in the field believe that invasive diagnostic procedures should be resorted to. First, duodenal juice should be sampled and examined for the presence of $G$. lamblia trophozoites, and then histopathological examination of the digestive tract mucosal (primarily duodenal) biopsies should be done. Endoscopy has markedly improved the diagnosis in the practice of internal medicine, allowing at the same time parasitological examination for the presence of G. lamblia parasite $[7,8,20,21]$.

In our own study, in addition to CM of three stool samples with and without the concentration method and EIA test to detect G. lamblia antigen in one stool sample, endoscopic examination was done in all hospitalized patients, with sampling of the duodenal juice for parasitological analysis, and stomach and duodenal mucosa biopsies for histopathological analysis.

G. lamblia trophozoites in the duodenal juice were established in only 2 of 4 adult patients with confirmed giardiasis. Examination of the biopsies yieled even smaller number of positive findings. In only one female patient, in whom the presence of G. lamblia was established using other methods as well, the studied protozoan was found in the duodenal mucosa on histological preparations. Some authors have stated that the sensitivity of invasive methods, i.e. parasitological examination of the duodenal juice and biopsies of the duodenal mucosa for histopathological analysis, is only 44\% [22]. Low sensitivity, especially of parasitological examination of the duodenal juice as that obtained in our study, can be explained by the fact that microscopy of duodenal juice preparations is not performed during or immediately after endoscopy, nor it is performed at the same facility (building) with endoscopy. Local unavailability of parasitology laboratories and inability of G. lamblia to survive transport to the lab could be one of the causes of small number of positive findings. Moreover, parasitological examination for the presence of G. lamblia trophozoites in the duodenal juice has not been a part of the regular, routine work of the endoscopy departments, and it is possible that the material has been inadequately or with delays transported to the parasitology lab. These circumstances could have influenced the results showing low sensitivity $(50 \%)$ of the method of parasitological examination of the duodenal juice for the presence of G. lamblia parasite.

The finding of $G$. lamblia in only one biopsy sample did not differ from other authors' findings. In many studies aiming to confirm the presence of the protozoan on or in the mucosa of the duodenum, the obtained results have shown that even when the protozoan is present in the bowel lumen, at the same time it is not found in the histological preparations of duodenal mucosa [20,21].

In our study, it was established that all patients with confirmed G. lamblia presence had a varying degree of damage to the duodenal and gastric mucosa. However, since in all hospitalized patients with diagnosed giardiasis, parasitic infection was found and internistic diagnosis was made as well, it was difficult to attribute the damage to the duodenal and gastric mucosa only to pathogenetic mechanisms related to the presence of G. lamblia. All the patients with giardiasis (regardless of the method used to establish its presence) had a histopathological finding, but all hospitalized patients with histopathological findings of their gastric and duodenal mucosa were not infected with the protozoan. In only one female patient developed chronic atrophic grade II duodenitis was established, and G. lamblia trophozoites were found in the mucus, so it could be assumed that the protozoan had a role in the pathogenesis of damage to the duodenal mucosa. In other patients with giardiasis confirmed using some of the available diagnostic procedures, histopathological findings showed chronic atrophic changes to the duodenal mucosa of a milder severity (I and II grade), as well as chronic atrophic grade II gastritis. All the presented patients had the initial, working diagnosis of dyspeptic syndrome. After the parasite G. lamblia was confirmed using the above diagnostic procedures, the final etiological diagnosis of a digestive tract infection (giardiasis) was made.

\section{Conclusions}

From our results a conclusion may be drawn that invasive methods do not have sufficient sensitivity in the diagnosis of giardiasis, if the presence of factors of influence on false negative results is excluded, as well as the so called „transit" through the digestive tract, when the parasites can be detected in the stool, without being present in the digestive tract or in the biopsy samples. In view of a high prevalence of giardiasis in the Nišava District (southeastern Serbia), the need should be stressed that each duodenal juice sample should be examined for the presence of $G$. 
lamblia parasite, and that this should become a regular procedure after each endoscopy. Histopathological examination is the sole procedure that can resolve the dilemma of the magnitude of role of G. lamblia in the onset of damage to the digestive tract, primarily duodenal, mucosa. Non-invasive, standard diagnostic parasitological procedures in the diagnosis of giardiasis remain as the methods of choice, while the EIA method should be preferred for screening purposes in epidemiological studies.

\section{References}

1.GARZIA LS, SHIMIZU RY. Detection of Giardia lamblia and Cryptosporidium parvum antigens in human fecal specimens using the ColorPac combination rapid solid-phase qualitative immunochromatographic assay. J Clin Microbiol 2000, 38:1267-1268

2.GARCIA LS, SHIMIZU RY. Evaluation of nine immunoassay kits (enzyme immunoassay and direct fluorescence) for detection of Giardia lamblia and Cryptosporidium parvum in human fecal specimens. J Clin Microbiol 1997, 35:1526-1529

3.MANK TG, ZAAT JO, DEELDER AM, VAN EIJK JT, POLDERMAN AM. Sensitivity of microscopy versus enzyme immunoassay in the laboratory diagnosis of Giardiasis. Eur J Clin Microbiol Infect Dis 1997, 16:615-619

4.ROSOFF JD, SANDERS CA, SEEMA SS, DeLAY PR, HADLEY WK, VINCENZI FF, YAJKO DM, O'HANLEY PD. Stool diagnosis of Giardiasis using a commercially available enzyme immunoassay to detect Giardia-specific antigen 65(GSA 65). J Clin Microbiol 1989, 27:19972002

5.BEHR AM, KOKOSKIN E, GYORKOS WT, CÉDILOTTE L, FAUBER MG, and MACLEAN DJ. Laboratory diagnosis for Giardia lamblia infection: a comparison of microscopy, coprodiagnosis and serology. Can J Infect Dis 1997, 8:33-38

6.NATAŠA MILADINOVIĆ TASIĆ, SUZANA A. TASIC, IVAN S. TASIC. Modern diagnostics of giardiasis. Acta Fac Med Naiss 2008, 25(2): 97-101

7.HILL DR. Giardiasis: Issues in management and treatment. Infect Dis Clin North Am 1993, 7:503-525

8.FARTHIN MJG. Giardiasis. Gastroenterol. Clin North Am 1996, 25:493-515

9.CACCIO SM, RYAN U. Molecular epidemiology of giardiasis. Molecular\&Biochemical Parasitology 2008, 160:75-80

10.THOMPSON RCA. Giardiasis: Modern Concepts in Control and Management. Annales Nestlé 2008, 66:23-29

11.PESTEHCHIAN N, RASEKH H, BABAEI Z, YOUSEFI HA, ESKANDARIAN AA, KAZEMI M, AKBARI M. Identification of genotypes of Giardia duodenalis human isolates in Isfahan, Iran, using polymerase chain reaction - Restriction Fragment Length polymorphism. Advanced Biomedical Research 2012, (8)1:84-90

12.ALEXANDER C., JONES B., INVERAROTY D., POLLOCKo KGJ. Genotyping of Giardia isolates in Scotland: a descriptive epidemiological stuy. Epidemiol Infect. 2014, 142:1636-1639

13.ANNUAR TS, AZREEN SN, SALLEH MdF, MOLTAR N. Molecular epidemiology of giardiasis among Orang Asli in Malaysia: application of the triosephosphate isomerase gene. BMC Infection Diseases 2014, 14:78

14.Kranjčić-Zec I, Mitrović S, Arsić AV, Đamić A. Medicinska parazitologija i mikologija, priručnik. Partenon, 1999.

15.WHITHEAD R. Mucosal biopsy of the gastrointestinal trakt. W. S. Saunders Company. Ltd., London, 1973, 1-46

16.KATIĆ V. Pathology of the stomach. Medical books•Belgrade-Zagreb, 1989, 84-128

17.LYNCH R. Medical laboratory technology and clinical pathology. Melor, Spare and Tuwood 1980.

18.LENGERICH EJ, ADDISS DG, JURANEK DD. Severe Giardiasis in the United States Clin Infect Dis 1994, 18:760-763

19.MARAHA B, BUITING AGM. Evaluation of Four Enzyme Immunoassays for the Detection of Giardia lamblia Antigen in Stool Specimens. Eur J Microbil Infect Dis 2000, 19:485-487

20.NASH TE ET AL. Experimental human infections with Giardia lamblia. J Infect Dis 1987, 156:974-984

21.OBERHUBER G, KASTNER N, STOLTE M. Giardiasis: a histologic analysis of 567 cases. Scand J Infect Dis 1997, 32:48-51

22.TESSIER JL, DAVIES GAL. Giardiasis. Primary Care Update for OB/GYNS 1999, 61(1):8-11

Manuscript received: 17.08 .2018 
